\title{
Candidate metastasis suppressor genes uncovered by array comparative genomic hybridization in a mouse allograft model of prostate cancer
}

\author{
Yajun $\mathrm{Yi}^{1}$, Srinivas Nandana ${ }^{2}$, Thomas Case ${ }^{2}$, Colleen Nelson ${ }^{3}$, \\ Tatjana Radmilovic ${ }^{1}$, Robert J Matusik ${ }^{2}$ and Karen D Tsuchiya*4,5
}

\begin{abstract}
Address: ${ }^{1}$ Department of Medicine, Vanderbilt University Medical Center, Nashville, TN, USA, ${ }^{2}$ Department of Urologic Surgery and Vanderbilt Ingram Cancer Center, Vanderbilt University Medical Center, Nashville, TN, USA, ${ }^{3}$ Department of Urologic Sciences, University of British Columbia, The Prostate Centre at Vancouver General Hospital, Vancouver, British Columbia, Canada, ${ }^{4}$ Clinical Research Division, Fred Hutchinson Cancer Research Center and Department of Laboratories, Seattle Children's Hospital, WA, USA and ${ }^{5}$ Department of Laboratory Medicine, University of Washington School of Medicine, Seattle, WA, USA

Email: Yajun Yi - andrew.yi@vanderbilt.edu; Srinivas Nandana - srinivas.r.nandana@vanderbilt.edu; Thomas Case - tom.case@vanderbilt.edu; Colleen Nelson - colleen.nelson@ubc.ca; Tatjana Radmilovic - tatjana_radmilovic07@yahoo.com;

Robert J Matusik - robert.matusik@vanderbilt.edu; Karen D Tsuchiya* - karen.tsuchiya@seattlechildrens.org

* Corresponding author
\end{abstract}

Published: 26 September 2009

Molecular Cytogenetics 2009, 2:18 doi:10.1186/1755-8166-2-18
Received: 6 April 2009

Accepted: 26 September 2009

This article is available from: http://www.molecularcytogenetics.org/content/2/1/18

(c) 2009 Yi et al; licensee BioMed Central Ltd.

This is an Open Access article distributed under the terms of the Creative Commons Attribution License (http://creativecommons.org/licenses/by/2.0), which permits unrestricted use, distribution, and reproduction in any medium, provided the original work is properly cited.

\begin{abstract}
Background: The purpose of this study was to identify candidate metastasis suppressor genes from a mouse allograft model of prostate cancer (NE-10). This allograft model originally developed metastases by twelve weeks after implantation in male athymic nude mice, but lost the ability to metastasize after a number of in vivo passages. We performed high resolution array comparative genomic hybridization on the metastasizing and non-metastasizing allografts to identify chromosome imbalances that differed between the two groups of tumors.

Results: This analysis uncovered a deletion on chromosome 2 that differed between the metastasizing and non-metastasizing tumors. Bioinformatics filters were employed to mine this region of the genome for candidate metastasis suppressor genes. Of the 146 known genes that reside within the region of interest on mouse chromosome 2, four candidate metastasis suppressor genes (Slc27a2, Mall, Snrpb, and Rassf2) were identified. Quantitative expression analysis confirmed decreased expression of these genes in the metastasizing compared to non-metastasizing tumors.

Conclusion: This study presents combined genomics and bioinformatics approaches for identifying potential metastasis suppressor genes. The genes identified here are candidates for further studies to determine their functional role in inhibiting metastases in the NE-I0 allograft model and human prostate cancer.
\end{abstract}

\section{Background}

Prostate cancer ( $\mathrm{PCa})$ is a heterogeneous disease and the ability to predict its clinical outcome is limited. Numerous chromosomal abnormalities and alterations in gene expression have been reported in $\mathrm{PCa}$, yet identification of many of the specific genes that drive the progression of these tumors is still lacking. The finding of the TMPRSS2/ ETS fusion and the overexpression of ETS transcription 
family members in the majority of PCa illustrates the success of utilizing a bioinformatics approach to gene discovery [1], but the consequences of many other recurrent acquired genomic alterations remain to be elucidated. Studies of human PCa are hindered by the biologic and genetic heterogeneity of this disease not only between individuals, but also within a given individual. Genetically engineered mouse models of PCa provide an in vivo experimental system in which tumors with the same underlying etiology can be sampled during the course of progression at defined time points. The LPB-Tag mouse model of prostate cancer is one such model that has been well characterized [2-4].

We have previously described the establishment of an allograft (NE-10) from a primary prostate tumor from the LPB-Tag mouse model that consistently metastasized by 12 weeks after transplantation in nude mice in early passages [4]. Conventional cytogenetic analysis of the NE-10 allograft revealed numeric and structural chromosome abnormalities, including a deletion of distal chromosome 2 that was consistently present over multiple in vivo passages of the allograft in nude mice [4]. A similar chromosome 2 deletion has also been described in a mouse model of acute promyelocytic leukemia [5]. After repeated in vivo passages, the NE-10 allograft eventually lost the ability to metastasize. In this study, we have taken advantage of the differential metastasizing behavior of the NE10 allograft, arising from the same original tumor, to screen the genome for candidate genes that play a role in metastasis. High resolution genomic technology, combined with novel bioinformatics approaches, enabled us to identify different regions of chromosome imbalances between the two allograft lines, and to propose candidate metastasis suppressor genes within a region of chromosome 2 that was found to harbor a larger deletion in metastatic compared to non-metastatic tumors.

\section{Methods}

\section{NE-10 allograft model}

The 12T-10 line of the LPB-Tag mouse model of prostate cancer was generated using a transgene that consists of the rat probasin promoter driving the SV40 large $\mathrm{T}$ antigen with deletion of the small $\mathrm{T}$ antigen [3]. These mice develop low-grade and high-grade prostatic intraepithelial neoplasia at 2-5 month of age, with progression to invasive and metastatic, high-grade, androgen-independent carcinoma demonstrating neuroendocrine differentiation at 6-14 months of age. A primary prostate tumor from the ventral prostate of a 12T-10 transgenic mouse was used to establish an allograft model by implantation subcutaneously in male athymic nude mice [4]. After 18 weeks, the allograft was passaged to another male nude mouse and the process was repeated to establish the NE10 line [4]. Initial passages in all mice developed grossly visible metastases to liver and micrometastases to lung by twelve weeks after implantation. All metastases from the allografts were histologically similar to the metastasis seen in the 12T-10 mice. Later allograft passages showed histologically identical features to the early passages; however, fewer allografts developed metastases. By passage 15, metastatic potential was completely lost, at least up to the point where it was no longer feasible to maintain the mice due to the size of the subcutaneous tumors. Tumors consisting of non-metastasizing subcutaneous allografts (SQnon-met), metastasizing subcutaneous allografts (SQmet), and liver metastases (LiverMet) were collected at 12 weeks post-implantation and snap-frozen for array CGH. All procedures involving mice were approved by the Vanderbilt University Medical Center and Fred Hutchinson Cancer Research Center Institutional Animal Care and Use Committees.

\section{Array comparative genomic hybridization (CGH)}

Array CGH was initially carried out using mouse bacterial artificial chromosome (BAC) arrays produced by the Genomics Shared Resource, Fred Hutchinson Cancer Research Center as described [6]. The mouse BAC clone set was obtained from A. Bradley, Wellcome Trust Sanger Institute [7] and provides an average resolution of $1 \mathrm{Mb}$. DNA was isolated using the Gentra Puregene genomic DNA purification kit (Qiagen, Valencia, CA). For the BAC arrays, three SQnon-met tumors (passage 19), three SQmet (passages 4, 9, and 12), and four LiverMet (two each from passages 9 and 12) were analyzed. One matched SQmet and LiverMet from the same nude mouse at passage 9 was included in the analysis. Pooled DNA obtained from normal kidney from four different CD-1 males was used as a reference. DNA labeling, hybridization, scanning, and data analysis was performed as described previously [6].

Array CGH was repeated on a subset of two tumors each from the SQnon-met and LiverMet groups using the Agilent mouse $105 \mathrm{~K}$ oligonucleotide CGH arrays. These arrays were designed using UCSC mm7 (NCBI build 35, August, 2005), and have an average probe spacing of 15 $\mathrm{Kb}$. One ug of RsaI/AluI digested DNA was labeled with either Cy3 or Cy5 using the BioPrime Array CGH genomic labeling system (Invitrogen Corp., Carlsbad, CA). Approximately 4 ug each of Cy5 labeled test (tumor) DNA and Cy3 labeled reference (normal female C57Bl/6 liver) DNA was combined with 25 ug of mouse Cot-1 (Invitrogen Corp.), Agilent $10 \times$ blocking agent and $2 \times$ hybridization buffer (Agilent Technologies, Santa Clara, CA) to a final volume of $260 \mathrm{ul}$. Hybridization and washes were carried out according to the Agilent oligo array-based CGH protocol v. 4.0. Scanning was performed on an Agilent scanner and data extraction was carried out using Agilent feature extraction software v.9.1, employing linear and Lowess normalization. Results were analyzed and chromosome plots generated using CGHanalytics software v. 6.0. The Z- 
score algorithm with the threshold set at 2.0 and a $2 \mathrm{Mb}$ window was used for determining gains and losses. The LPB-Tag tumors were generated on an outbred CD-1 background, and we did not have non-neoplastic DNA available from the mouse whose tumor was used to establish the allograft. As $\mathrm{C} 57 \mathrm{Bl} / 6$ reference DNA was used for the oligonucleotide array CGH experiments, gains and losses smaller than $2 \mathrm{Mb}$ were ignored in order to avoid mistaking benign copy number variants between mouse strains from acquired copy number alterations in the tumors.

To compare array CGH profiles between the different groups of tumors, the Differential Gene locus MAPping software (DIGMAP version 2.0) was used to analyze array CGH data sets [8]. Using the default parameters specified in the program, DIGMAP displayed array CGH data as a heat map based on $\log _{2}$ ratio between test and reference DNA for each tumor. The chromosome regions with significantly different $\log _{2}$ ratios were marked as differential flagged regions (DFRs) by direct visualization and computational screening using a T-test based sliding window (TTSW) analysis. For this analysis, a window size of 40 genes was used and DFRs represent regions greater than 3 standard deviations from the whole genome average $\mathrm{T}$ score.

\section{Expression arrays}

Expression arrays were performed on two SQnon-met tumors (passages 11b, 18c) and four SQmet tumors (passages 2, 5, 14a, 14c). Allograft passages were labeled a, b, $c$, etc. when the same graft passage was implanted into more than one mouse. As a control, mouse reference RNA from two postnatal day 1 mice was pooled. RNA was isolated using the RNeasy kit (Qiagen Valencia CA), including treatment with DNase. RNA was quantified using a NanoDrop (ThermoScientific). All samples analyzed had a A260/230 ratio greater than 1.8. RNA quality was analyzed on an Agilent 2100 BioAnalyzer. All samples analyzed had a $28 \mathrm{~S}$ peak greater than $18 \mathrm{~S}$ peak, or an RIN number greater than 7 . The $16 \mathrm{k}$ mouse arrays used for the study were printed at the Vancouver Microarray Facility using Operon's 16 K 70 mer oligomers printed on aminosaline slides. Ten ug of total RNA was labeled using Genisphere's Dendrimer 350 expression array detection kit for microarrays according to the manufacturer's protocol. Samples were co-hybridized with ten ug of the above described mouse reference RNA. Arrays were pre-hybridized in $5 \times$ SSC, $0.1 \%$ SDS and $0.2 \%$ BSA for 45 minutes. Pre-hybridization buffer was washed off with $3 \times 30 \mathrm{sec}$ ond water washes and a 2 minute wash in isopropanol. Slides were spun dry at $2000 \mathrm{rpm}$ for 4 minutes. The samples were applied to arrays containing $60 \times 22 \mathrm{~mm}$ lifter slips (Erie Scientific). Slides were subsequently treated as described in the Genisphere Array 350 expression array detection kit for microarrays. Arrays were scanned on a Axon 4200AL scanner (Molecular Devices). Image inten- sities were extracted using ImaGene V8.0 software (BioDiscovery).

To identify chromosome regions that demonstrate differential gene expression between metastatic and non-metastatic tumors, expression array datasets were analyzed between SQnon-met and SQmet mice using DIGMAP as described above for array CGH data. A TTSW genome scan was also performed as described above.

\section{Identification of candidate metastasis suppressor genes in chromosome 2 DFR}

A total of 146 known genes in the chromosome 2 differential region of deletion (DFR) between metastatic and non-metastatic tumors (nucleotides 122,316,740$139,585,560)$ were retrieved from the UCSC mm7 database http://genome.ucsc.edu. Two bioinformatics filters were designed to determine which of these 146 genes might function as candidate metastasis suppressor genes. A functional filter utilized the Gene Ontology (GO) database http://www.geneontology.org, PubMed http:// www.ncbi.nlm.nih.gov/PubMed/, and Ingenuity Pathway Analysis (IPA, http://www.ingenuity.com) to evaluate the 146 genes for potential metastasis suppressor function. A parallel filter employing our recently developed human cancer expression signature database (EXALT) was used to independently validate the genes as candidate metastasis suppressors in silico [9]. A weighted score was assigned to each gene for both filters (see Additional file 1).

\section{Quantitative reverse transcription (RT)-PCR}

Total RNA was isolated using the RNeasy Mini kit (Qiagen, Valencia, CA) according to the manufacturer's protocol, including the recommended DNase treatment step. Three ug of RNA was reverse transcribed using the ReactionReady First Strand cDNA Synthesis Kit (SuperArray Bioscience Corp., Frederick, MD). Primer sets for mouse Hprt1, Slc27a2, Mal, Snrpb, and Rassf2 were purchased from SuperArray (proprietary primers, sequence not disclosed).

PCR reactions were carried out in triplicate in a $25 \mu \mathrm{L}$ volume using SYBR Green Master Mix (SuperArray). The standard two-step amplification with an annealing temperature of $60^{\circ} \mathrm{C}$ was performed in an ABI 7900 PCR machine. Hprt1 was chosen as an endogenous control gene because Hprt1 expression values were stable among test samples in the microarray expression data set.

To allow for a comparison between samples and the two groups, quantities of all target genes in the test samples and a common reference RNA (Mouse XpressRef Universal Total RNA, SuperArray) were normalized to the corresponding Hprt1 levels. Relative expression levels (fold changes) were calculated using the relative standard curve method as outlined in the manufacturer's technical user 
manual (SuperArray). A standard curve was generated using the fluorescent data from 10-fold serial dilutions of the common reference RNA sample. Four tumors each from the metastasizing and non-metastasizing allografts were analyzed. Statistically significant differences in expression between the two groups were determined using a Student's T-Test.

\section{Results \\ Differences in copy number alterations between metastatic and non-metastatic tumors}

Copy number alterations in tumors were identified by array CGH performed using BAC arrays for all tumors, and oligonucleotide arrays for a subset of tumors. Array CGH using both BAC and oligonucleotide platforms detected multiple gains and losses in both non-metastatic tumors (SQnon-met) and tumors with metastatic potential (SQmet and LiverMet). Both array platforms uncovered similar chromosome imbalances, but the oligonucleotide arrays allowed us to more precisely map regions of gain and loss. Many copy number alterations were common to both groups of tumors, including deletions involving regions of chromosomes $1,2,3,4,8,13$, 16, 1718,19 , whole chromosome loss of 14 , and gains of chromosome 8 (Figure 1 and Table 1); however, differences in copy number changes between the tumors with and without metastatic potential were also uncovered (Figure 1 and Table 1). One notable difference between the two groups of tumors was a differential region of deletion in distal chromosome 2. Both the metastatic and non-metastatic tumors shared a common $27.4 \mathrm{Mb}$ interstitial deletion of distal chromosome 2, from bands F3 to H3 (139,585,560-167,088,181 bp); however, the metastatic tumors (LiverMet) showed a larger $45 \mathrm{Mb}$ deletion $(122,316,740-167,088,181 \mathrm{bp})$ that extended proximally to band E5 (Figure 2). The non-metastatic tumors showed clonal heterogeneity for both the larger and smaller deletions, with low-level mosaicism for the larger deletion (Figure 2 top), but the clone or clones with the larger deletion were enriched in the metastatic tumors (Figure 2 bottom).

\section{Differences in gene expression profiles between metastatic and non-metastatic tumors}

To obtain differential gene expression profiles between non-metastatic and metastatic NE-10 tumors, the different groups of tumors (SQnon-met, SQmet, and LiverMet) were profiled using gene expression microarrays and analyzed by DIGMAP. A T-test sliding window whole genome scan was performed and plotted by chromosome (see Additional file 2). Based on this analysis, chromosome 2 had the greatest number of regions showing the largest difference in gene expression between metastatic (SQmet and LiverMet) and non-metastatic (SQnon-met) tumors. A detailed T-test sliding window plot of chromosome 2 revealed the highest DFR expression peak in band H4,

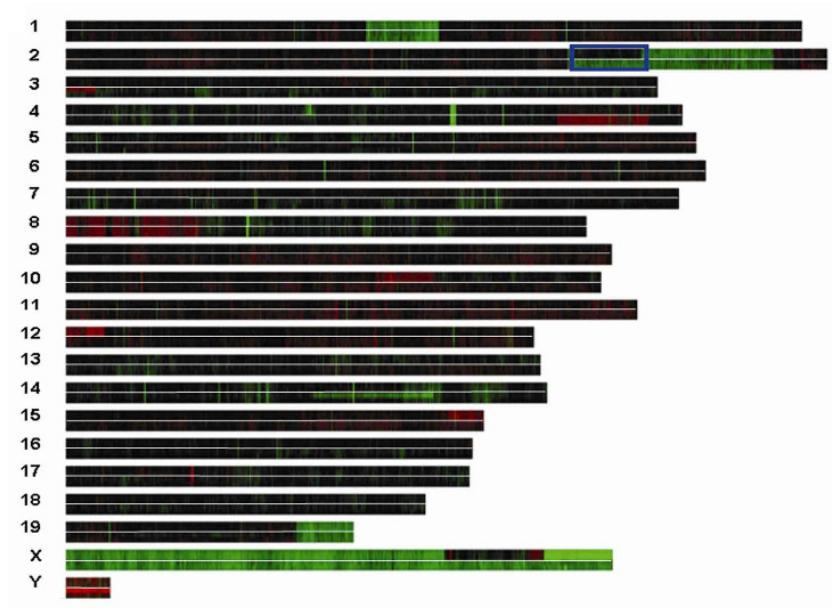

Figure I

Heat map of oligonucleotide array CGH results.

Array CGH results are partitioned by chromosome number and clustered by probe location in the chromosomes. The length of each chromosome bar is based on the total number of probes for that chromosome present on the array. Within each chromosome bar, each row represents a separate NE10 tumor, and the non-metastatic tumors (SQnon-Met) are separated from the metastatic tumors (LiverMet) by the white line (SQnon-Met above and LiverMet below the line). Black represents normal copy number in tumor compared to the normal reference; red represents increased copy number; green represents decreased copy number. A larger interstitial deletion in chromosome 2 can be visualized in the metastatic compared to the non-metastatic tumors (blue box). Relative loss of the $X$ chromosome in the metastatic tumors, and most of the $X$ chromosome in the non-metastatic tumors, is seen because array CGH for these tumor samples was performed using a sex-mismatched (female) reference DNA. The non-metastatic tumors also show a region of copy number gain on the $\mathrm{X}$ chromosome.

from 179 to $181 \mathrm{Mb}$ (see Additional file 2). This region is distal to the common region of deletion found in both the metastatic and non-metastatic tumors. Three other smaller peaks were also located slightly more centromeric, from 155 to $168 \mathrm{Mb}$, within the common region of deletion. Thus, regions of differential gene expression between metastatic and non-metastatic tumors were located both within and distal to the common chromosome 2 deletion; however, we were unable to detect any clusters of genes that demonstrated down-regulation in the metastatic compared to the non-metastatic tumors. There were also no significant differences in gene expression detected in the differential region of deletion on chromosome 2 between the metastatic and non-metastatic tumors.

\section{Identification of candidate metastasis suppressor genes in chromosome 2 differentially deleted region}

Metastasis suppressor genes are expected to show inactivation or decreased activity in metastatic tumors because of 

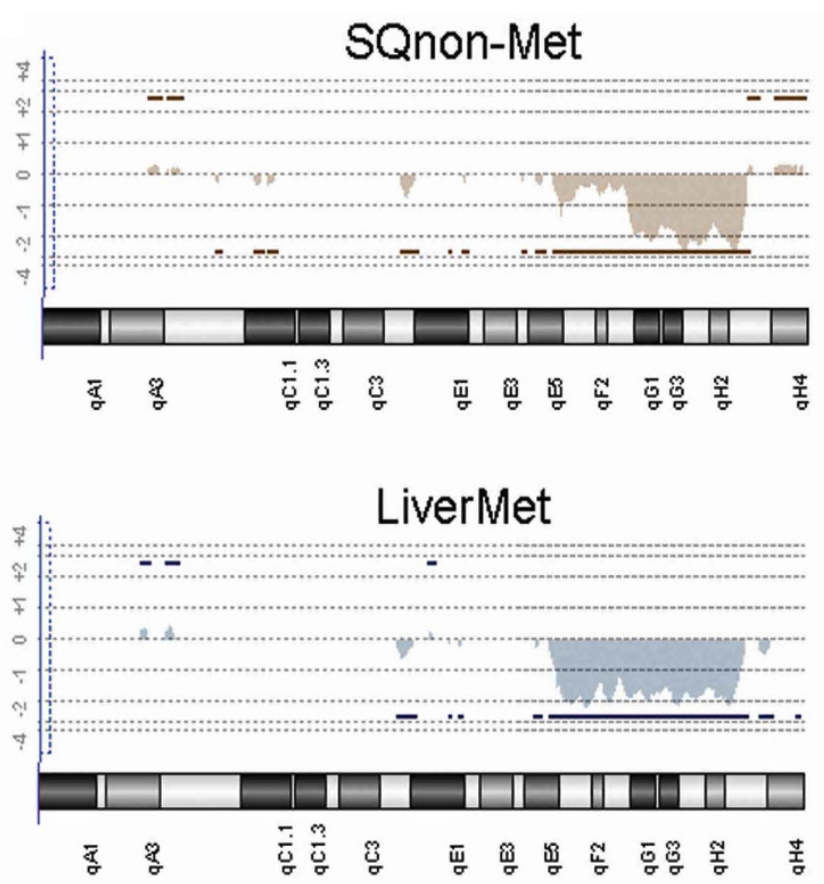

Figure 2

Chromosome 2 oligonucleotide array CGH plots. Chromosome 2 plots are shown for one representative nonmetastatic (SQnon-Met) and one representative metastatic (LiverMet) tumor. The normalized log2 ratio is on the $Y$ axis, and the chromosome bands are designated on the $X$ axis. Copy number gains and losses are designated by the bars above or below a log 2 ratio of 0 , respectively. The shaded area reflects the magnitude of the gain or loss. The metastatic tumors show only the larger chromosome 2 deletion (bottom panel), whereas the non-metastatic tumors demonstrate clonal heterogeneity, with the both the smaller deletion, as well as low-level mosaicism for the larger deletion (top panel).

genetic or epigenetic changes that result in loss or downregulation of expression. The finding of a differentially deleted region on chromosome 2 , potentially resulting in loss of a metastasis suppressor gene in the metastatic tumors, led us to focus on this region for more detailed analysis. Given that only a subset of genes in the differentially deleted region was represented on the expression arrays, we employed an informatics approach to identify all potential candidate metastasis suppressor genes in the region of interest (chromosome 2 E5-F3; nucleotides $122,316,740-139,585,560)$. Two parallel bioinformatics filters were designed to carry out this analysis. Out of a total of 146 genes in the region of interest, 11 candidate metastasis suppressor genes were identified based on the function-based filters, while 22 candidates were identified using a filter that employed a cancer expression signature
Table I: Summary of acquired copy number changes identified by oligonucleotide array CGH in liver metastases (LiverMet) from NE- 10 allografts, and in non-metastasizing (SQnon-met) NE-I 0 allografts

\begin{tabular}{|c|c|c|}
\hline Chromosome & SQnon-met & LiverMet \\
\hline I - loss & $* 84525268-103082423$ & $84525268-103082423$ \\
\hline 2 - loss & $|39585560-| 67088|8|$ & $|22316740-167088| 8 \mid$ \\
\hline 3 - loss & II809017-qter & 11809017-qter \\
\hline 4 - loss & $3010281-131339250$ & $3010281-131339250$ \\
\hline 4 - gain & N.A. & | 32282308-| 49233400 \\
\hline 5 - loss & $3003879-114937488$ & N.A. \\
\hline 7 - loss & N.A. & whole chromosome \\
\hline 8 - gain & $8397754-17345 \mid 26$ & $8397754-17345 \mid 26$ \\
\hline 8 - gain & $20825979-35687899$ & $20825979-35687899$ \\
\hline 8 - loss & 37627333-qter & 37627333-qter \\
\hline 10 - gain & $79500370-89346058$ & N.A. \\
\hline 10 - loss & 89748171 -qter & N.A. \\
\hline 12 - gain & $3021012-12590005$ & N.A. \\
\hline 13 - loss & whole chromosome & $3015154-60624834$ \\
\hline 14 - loss & whole chromosome & whole chromosome \\
\hline 15 - gain & 99348507-qter & N.A. \\
\hline 16 - loss & $3026317-36861999$ & whole chromosome \\
\hline 17 - loss & $3023355-10795310$ & whole chromosome \\
\hline 17 - loss & 29138819 -qter & whole chromosome \\
\hline 18 - loss & 35576728-qter & whole chromosome \\
\hline 19 - loss & 4697I550-qter & 4697I550-qter \\
\hline$X$ - gain & $|45268084-| 49|| 4686$ & N.A. \\
\hline
\end{tabular}

N.A. = not applicable

*Nucleotide sequences are from NCBI build 35

filter using the EXALT database (see Additional file 3). A final list of four candidate genes (Slc27a2, Mall, Snrpb, and Rassf2) was chosen based on having the highest total score. 
Quantitative RT-PCR of 4 candidate metastasis suppressor genes in chromosome 2 differentially deleted region

Quantitative RT-PCR for the four candidate metastasis suppressor genes was performed in tumors with metastatic potential (SQMet) and tumors without metastatic potential (SQnon-Met). Metastatic liver tumors were not analyzed due to the presence of contaminating normal liver tissue in some of the tumors. All four candidate metastasis suppressor genes demonstrated decreased expression in the metastasizing tumors compared to the non-metastasizing tumors, with Slc27a2 and Snrpb showing statistically significant decreased expression in the metastasizing tumors (Figure 3).

\section{Discussion}

Using high-resolution array $\mathrm{CGH}$, we uncovered multiple copy number changes common to both the metastatic and non-metastatic tumors, consistent with a phenotype of genomic instability in this model. Many of the regions of loss overlap with frequent losses that have been observed by conventional CGH and array CGH performed on localized and metastatic human PCa, including human 4q22.3-q31.1, 5q21.1-q21.3, 6q15-q16.2,

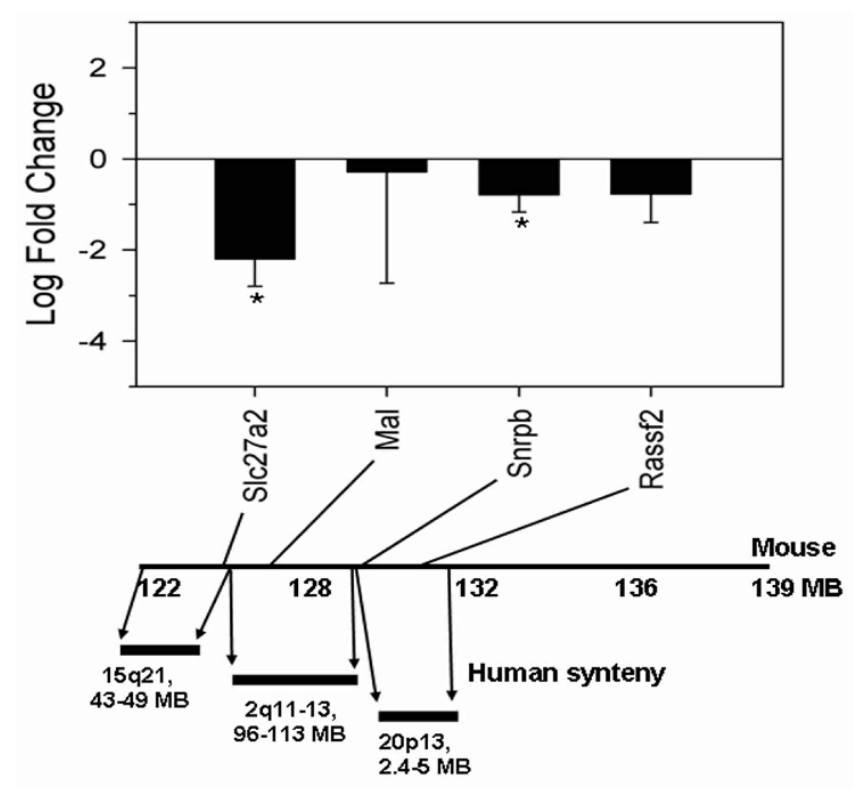

Figure 3

Relative expression levels between metastatic and non-metastatic NE- 10 tumors by RT-PCR. The $X$-axis shows four candidate metastasis suppressor genes and the $Y$ axis shows relative fold change (log2 based) between SQMet and SQnonMet NE-I0 tumors (top panel). The relative expression levels are designated by the height of bars, and standard deviation error bars are in the direction of SQMet samples. An asterisk indicates significantly decreased expression in SQMet samples $(P<0.05)$. The corresponding genomic locations of the four candidate genes on mouse chromosome 2 , and the regions of conserved synteny in human, are shown below. 8p21.1-p23.1, 10q23.1, 10q25.1-q25.3, 13q14.2-q22.1, 16q12.1-q24.3, 18q12.3-q21.1, 18q21.31-q21.32, 18q22.1-q23 [10-12]. Loss of specific tumor suppressor genes implicated in human PCa, such as Nkx3-1 and $R b 1$, was also present in the mouse NE-10 allograft. Other alterations that are frequently seen in human $\mathrm{PCa}$, such as loss of PTEN, loss of CDKN1B, and gain of MYC, were not observed in the NE-10 model.

In addition to copy number alterations common to both the metastatic and non-metastatic tumors, we also observed differences in copy number alterations between these two groups of tumors that could be responsible for their divergent behavior. These differences included loss of chromosome 2 (122.3-139.6 Mb), gain of chromosome 4 (132.2-149.2 Mb), loss of chromosome 7, loss of chromosome 16 (36.9 Mb -qter), loss of chromosome 17 (10.8- 29.1 Mb), and loss of chromosome 18 (3.3-35.6 $\mathrm{Mb})$ in the metastatic, but not the non-metastatic tumors. Our attention was drawn to a differentially deleted region of chromosome 2 for a number of reasons. First, haploinsufficiency or inactivation of metastasis suppressor genes can occur through deletion. We have also detected distal chromosome 2 deletions in approximately 35\% of metastatic tumors from multiple independent mice from the original 12T-10 transgenic line (unpublished data), confirming that this is a recurring chromosome abnormality in tumors from this model. In addition, a similar deletion of chromosome 2 has previously been reported in a mouse model of acute promyelocytic leukemia [5]. Also, the larger region of deletion that is enriched in the metastatic tumors demonstrates conserved synteny with the short arm of human chromosome 20 that has been implicated in metastatic PCa [13]. In the mouse model of acute promyelocytic leukemia, loss of one copy of the Sfpi1 (Pu.1) gene due to the chromosome 2 deletion, and reduced expression of this gene, have been implicated in the progression of leukemia in these mice [14]. The Sfpi1 gene is not an obvious candidate metastasis suppressor gene in the NE-10 allograft model, as the gene maps approximately $31 \mathrm{Mb}$ centromeric to the proximal chromosome 2 deletion breakpoint in the metastatic tumors. Cd82 (Kai1) and Cd44, known metastasis suppressor genes for prostate $[15,16]$, are also located many megabases ( $29 \mathrm{Mb}$ and $20 \mathrm{Mb}$, respectively) proximal to the chromosome 2 deletion. Expression array analyses that we performed also did not uncover candidate metastasis suppressor genes within this differentially deleted region; therefore, we employed a bioinformatics approach to identify potential candidate genes that may be involved in the metastatic behavior of these tumors.

The candidate metastasis suppressor genes in the differential region of deletion of chromosome 2 that we identified, based on prior evidence of metastasis suppressor function and down-regulation in cancer, consisted of 
Slc27a2, Mall, Snrpb, and Rassf2. All of these genes demonstrated decreased expression in the metastasizing compared to the non-metastasizing NE-10 tumors by quantitative RT-PCR. MALL (MAL, T-cell differentiation protein) is a raft-associated integral membrane protein that is involved in membrane trafficking processes. Expression of the MAL protein has been demonstrated in specific types of normal epithelial cells throughout the respiratory system, the gastrointestinal tract, and the genitourinary tract, including strong expression in the ductal and acinar cells of the prostate [17]. Loss of or decreased MALL expression, sometimes as the result of DNA methylation of the promoter region, has been found in a variety of benign and malignant epithelial tumors compared to their normal epithelial counterparts, consistent with a role for this protein in tumor suppression [17-21]. Furthermore, MAL has been shown to enhance apoptosis through the Fas pathway, and suppress tumorigenicity, invasion, and motility [21]. Although loss or decreased $M A L L$ expression has not been evaluated in human prostate cancer, the expression of this protein in normal prostate epithelium and its ability to suppress invasion and motility render it a biologically plausible metastasis suppressor gene for prostate cancer.

SLC27a2 encodes a protein that is an isozyme of the longchain fatty-acid-coenzyme A ligase family, and as such plays a role in lipid biosynthesis and fatty acid degradation. It may also be involved in translocation of longchain fatty acids across membranes [22]. Long-chain fatty acids participate in many cellular functions and have been implicated as modulators of carcinogenesis, partly through their ability to activate peroxisome proliferatoractivated receptors (PPAR). There is evidence that PPAR gamma regulates prostatic epithelial differentiation and may restrict epithelial proliferation; therefore, it is possible that decreased expression of Slc27a2 in the NE-10 allograft model could alter the tumor suppressor activity of PPAR gamma and contribute to metastatic behavior.

With regard to RASSF2 and SNRPB, Goodzari and coworkers found evidence for a prostate cancer metastasis suppressor gene on the short arm of human chromosome 20 [13]. The differential region of chromosome 2 deletion in metastatic compared to non-metastatic tumors in the NE-10 model shows conserved synteny with a region of human 20p. RASSF2 and SNRPB also map to the short arm of human chromosome 20, although they are telomeric to the most likely metastasis suppressor region of 20p (20p11.23-p12) that was proposed [13]. SNRPB encodes a protein that is one of several nuclear proteins that are found in common among U1, U2, U4/U6, and U5 small ribonucleoprotein particles (snRNPs). These snRNPs are involved in pre-mRNA splicing. RASSF2 (RAS association domain family 2) is a negative effector of Ras and has been implicated as a tumor suppressor gene in a number of other forms of epithelial cancer, such as colon, gastric, breast, and lung carcinoma [23-25]. The mechanism of inactivation of RASSF2 described in these tumors is aberrant promoter methylation, primarily in early tumors. In addition, inactivation of the A isoform of RASSF2 by promoter methylation has been found to correlate with a higher frequency of lymph node metastases in patients with nasopharyngeal carcinoma [26].

In summary, using an approach based on bioinformatic filters applied to array CGH data on a divergent metastasizing and non-metastasizing mouse model of PCa, we have identified genes that are biologically plausible metastasis suppressor genes. Our studies have identified an association between deletion with decreased expression of these genes and the metastasizing NE-10 model, suggesting a biological role for these genes in the metastatic process. We believe the divergence in behavior of the allograft can be attributed to clonal heterogeneity, with both metastasizing and non-metastasizing clones present in early allograft passages, but with selection for a nonmetastasizing clone or clones after multiple in vivo passages. This selection could be due to preferential subcutaneous growth of the non-metastasizing compared to the metastasizing component of the allograft, such that over time, a larger and larger percentage of the allograft consists of the non-metastasizing population. It is possible that the clone or clones with the larger chromosome 2 deletion are enriched in the metastatic tumors because the differentially deleted region itself is responsible for the metastatic behavior, either alone or in combination with other genetic alterations, or that the larger deletion is simply a marker for a clone that is selected for because of the presence of some other alteration that confers metastatic potential. Additional studies are needed to prove that a gene or genes within the differentially deleted region of chromosome 2 are responsible for suppression of metastatic behavior.

\section{Conclusion}

We have taken advantage of a mouse allograft model of $\mathrm{PCa}$ (NE-10) with divergent metastasizing and nonmetastasizing behavior to identify regions of the genome that potentially harbor metastasis suppressor genes. Using a combination of genomics and bioinformatics approaches, we identified candidate genes from a differentially deleted region on mouse chromosome 2 between the metastasizing and non-metastasizing allograft lines. The genes presented here are candidates for further studies to determine their functional role in inhibiting metastases in the NE-10 allograft model and human PCa.

\section{List of abbreviations}

NE-10: mouse allograft established from a primary tumor from the LPB-Tag mouse model of prostate cancer; PCa: prostate cancer; SQnon-met: non-metastasizing subcuta- 
neous allograft; SQmet: metastasizing subcutaneous allograft; LiverMet: liver metastases; CGH: comparative genomic hybridization; BAC: bacterial artificial chromosome; DFRs: differential flagged regions; TTSW: T-test sliding window.

\section{Competing interests}

The authors declare that they have no competing interests.

\section{Authors' contributions}

YY interpreted the expression array results, conducted the bioinformatics, directed and interpreted the RT-PCR experiments, and helped draft the manuscript. SN participated in the expression array experiments and helped revise the manuscript. TC maintained the allografts and harvested tissues. $\mathrm{CN}$ provided oversight of the expression array experiments. TR performed the quantitative RT-PCR experiments. RJM provided the allograft model, designed the expression array experiments, and helped draft the manuscript. KDT conducted and interpreted the array CGH experiments, and drafted the manuscript. All authors read and approved the final manuscript.

\section{Additional material}

\section{Additional file 1}

Weighted scoring for the selection of candidate genes in 2E5-2F3. Detailed description of the weighted scoring method that was used to select candidate suppressor genes in the differentially deleted region of chromosome 2 between metastasizing and non-metastasizing allografts.

Click here for file

[http://www.biomedcentral.com/content/supplementary/1755-

8166-2-18-S1.DOC]

\section{Additional file 2}

T-test sliding window whole genome and chromosome 2 analysis. Comparison of expression array results between metastatic and non-metastatic allografts using a $T$-test sliding window analysis.

Click here for file

[http://www.biomedcentral.com/content/supplementary/17558166-2-18-S2.PPT]

\section{Additional file 3}

Complete candidate gene list. Complete list of candidate genes from the 2E5-2F3 identified from the function-based bioinformatics filter and the cancer expression signature filter.

Click here for file

[http://www.biomedcentral.com/content/supplementary/17558166-2-18-S3.DOC]

\section{Acknowledgements}

We thank the Genomics Resource Core at the Fred Hutchinson Cancer Research Center for assistance with array CGH. This work was supported by $\mathrm{NIH}$ grants $\mathrm{NCl}$ ROI-CA76I42 (R.J.M.), DOD grant DAMD I7-03-I0035 (K.D.T.), NCl Howard Temin Award CAI I 4033 (Y.Y.), and the
Frances Williams Preston Laboratories of the T.J. Martell Foundation (R.J.M.).

\section{References}

I. Rubin MA, Chinnaiyan AM: Bioinformatics approach leads to the discovery of the TMPRSS2:ETS gene fusion in prostate cancer. Lab Invest 2006, 86: I099-I 102.

2. Kasper S, Sheppard PC, Yan Y, Pettigrew N, Borowsky AD, Prins GS, Dodd JG, Duckworth ML, Matusik RJ: Development, progression, and androgen-dependence of prostate tumors in probasinlarge $T$ antigen transgenic mice: a model for prostate cancer. Lab Invest 1998, 78:319-333.

3. Masumori N, Thomas TZ, Chaurand P, Case T, Paul M, Kasper S, Caprioli RM, Tsukamoto T, Shappell SB, Matusik RJ: A probasinlarge $T$ antigen transgenic mouse line develops prostate adenocarcinoma and neuroendocrine carcinoma with metastatic potential. Cancer Res 200I, 6 I:2239-2249.

4. Masumori N, Tsuchiya K, Tu WH, Lee C, Kasper S, Tsukamoto T, Shappell SB, Matusik RJ: An allograft model of androgen independent prostatic neuroendocrine carcinoma derived from a large probasin promoter-T antigen transgenic mouse line. | Urol 2004, I 7 I:439-442.

5. Zimonjic DB, Pollock JL, Westervelt P, Popescu NC, Ley TJ: Acquired, nonrandom chromosomal abnormalities associated with the development of acute promyelocytic leukemia in transgenic mice. Proc Natl Acad Sci USA 2000, 97:13306- I33 I I.

6. Loo LW, Grove DI, Williams EM, Neal CL, Cousens LA, Schubert EL, Holcomb IN, Massa HF, Glogovac J, Li Cl, et al.: Array comparative genomic hybridization analysis of genomic alterations in breast cancer subtypes. Cancer Res 2004, 64:854I-8549.

7. Chung YJ, Jonkers J, Kitson H, Fiegler H, Humphray S, Scott C, Hunt $\mathrm{S}, \mathrm{Yu}$ Y, Nishijima I, Velds A, et al.: A whole-genome mouse BAC microarray with I-Mb resolution for analysis of DNA copy number changes by array comparative genomic hybridization. Genome Res 2004, I 4: 188-196.

8. Yi Y, Mirosevich J, Shyr Y, Matusik R, George AL Jr: Coupled analysis of gene expression and chromosomal location. Genomics 2005, 85:40I-4I2.

9. Yi Y, Li C, Miller C, George AL Jr: Strategy for encoding and comparison of gene expression signatures. Genome Biol 2007, 8:RI33.

10. Sun J, Liu W, Adams TS, Li X, Turner AR, Chang B, Kim JW, Zheng $S L$, Isaacs WB, Xu J: DNA copy number alterations in prostate cancers: a combined analysis of published CGH studies. Prostate 2007, 67:692-700.

II. Lapointe J, Li C, Giacomini CP, Salari K, Huang S, Wang P, Ferrari M, Hernandez-Boussard T, Brooks JD, Pollack JR: Genomic profiling reveals alternative genetic pathways of prostate tumorigenesis. Cancer Res 2007, 67:8504-8510.

12. Kim JH, Dhanasekaran SM, Mehra R, Tomlins SA, Gu W, Yu J, KumarSinha C, Cao X, Dash A, Wang L, et al:: Integrative analysis of genomic aberrations associated with prostate cancer progression. Cancer Res 2007, 67:8229-8239.

13. Goodarzi G, Mashimo T, Watabe M, Cuthbert AP, Newbold RF, Pai SK, Hirota S, Hosobe S, Miura K, Bandyopadhyay S, et al.: Identification of tumor metastasis suppressor region on the short arm of human chromosome 20. Genes Chromosomes Cancer 2001, 32:33-42.

14. Walter MJ, Park JS, Ries RE, Lau SK, McLellan M, Jaeger S, Wilson RK, Mardis ER, Ley TJ: Reduced PU.I expression causes myeloid progenitor expansion and increased leukemia penetrance in mice expressing PML-RARalpha. Proc Natl Acad Sci USA 2005, I02:12513-12518.

15. Dong JT, Lamb PW, Rinker-Schaeffer CW, Vukanovic J, Ichikawa T, Isaacs JT, Barrett JC: KAII, a metastasis suppressor gene for prostate cancer on human chromosome IIpII.2. Science 1995, 268:884-886.

16. Yoshida BA, Chekmareva MA, Wharam JF, Kadkhodaian M, Stadler WM, Boyer A, Watabe K, Nelson JB, Rinker-Schaeffer CW: Prostate cancer metastasis-suppressor genes: a current perspective. In Vivo 1998, 12:49-58.

17. Marazuela M, Acevedo A, Adrados M, Garcia-Lopez MA, Alonso MA: Expression of MAL, an integral protein component of the machinery for raft-mediated pical transport, in human epithelia. J Histochem Cytochem 2003, 5 I:665-674. 
18. Lind GE, Ahlquist T, Lothe RA: DNA hypermethylation of MAL: a promising diagnostic biomarker for colorectal tumors. Gastroenterology 2007, 132:1631-1632. author reply I632

19. Mimori K, Nishida K, Nakamura Y, leta K, Yoshikawa Y, Sasaki A, Ishii $\mathrm{H}$, Alonso MA, Mori M: Loss of MAL expression in precancerous lesions of the esophagus. Ann Surg Oncol 2007, I4:1670-1677.

20. Hatta M, Nagai H, Okino K, Onda M, Yoneyama K, Ohta Y, Nakayama $\mathrm{H}$, Araki T, Emi M: Down-regulation of members of glycolipidenriched membrane raft gene family, MAL and BENE, in cervical squamous cell cancers. J Obstet Gynaecol Res 2004, 30:53-58.

21. Mimori K, Shiraishi T, Mashino K, Sonoda H, Yamashita K, Yoshinaga K, Masuda T, Utsunomiya T, Alonso MA, Inoue H, Mori M: MAL gene expression in esophageal cancer suppresses motility, invasion and tumorigenicity and enhances apoptosis through the Fas pathway. Oncogene 2003, 22:3463-347I.

22. Stahl A: A current review of fatty acid transport proteins (SLC27). Pflugers Arch 2004, 447:722-727.

23. Hesson LB, Wilson R, Morton D, Adams C, Walker M, Maher ER, Latif F: CpG island promoter hypermethylation of a novel Ras-effector gene RASSF2A is an early event in colon carcinogenesis and correlates inversely with K-ras mutations. Oncogene 2005, 24:3987-3994.

24. Endoh M, Tamura G, Honda T, Homma N, Terashima M, Nishizuka S, Motoyama T: RASSF2, a potential tumour suppressor, is silenced by CpG island hypermethylation in gastric cancer. BrJ Cancer 2005, 93:1395-1399.

25. Cooper WN, Dickinson RE, Dallol A, Grigorieva EV, Pavlova TV, Hesson LB, Bieche I, Broggini M, Maher ER, Zabarovsky ER, et al.: Epigenetic regulation of the ras effector/tumour suppressor RASSF2 in breast and lung cancer. Oncogene 2008, 27:|805-|8||.

26. Zhang Z, Sun D, Van do N, Tang A, Hu L, Huang G: Inactivation of RASSF2A by promoter methylation correlates with lymph node metastasis in nasopharyngeal carcinoma. Int J Cancer 2007, I 20:32-38.

Publish with Bio Med Central and every scientist can read your work free of charge

"BioMed Central will be the most significant development for disseminating the results of biomedical research in our lifetime. "

Sir Paul Nurse, Cancer Research UK

Your research papers will be:

- available free of charge to the entire biomedical community

- peer reviewed and published immediately upon acceptance

- cited in PubMed and archived on PubMed Central

- yours - you keep the copyright

Submit your manuscript here:

http://www.biomedcentral.com/info/publishing_adv.asp
BioMedcentral 